\title{
Dark Energy: Particle and Astronomical Examination
}

\author{
Nishant Kumar Sharma \\ S.B.S P.G. College Rudrapur, Uttrakhand, India \\ Author's Mail id:nkphysics11@gmail.com
}

Available online at: www.isroset.org

Received: 10/Jan/2020, Accepted: 20/Feb/2020, Online: 28/Feb/2020

\begin{abstract}
In This Manuscript We Described The Dramatic And Strange Behavior Of Particle And Matter Comes Under Dark Energy The Strange Behavior Is, That Holding The Properties To Interchange Their Conditions, And Varies Qualities Of Interchange Of Particles And Matter And Interchange With Mathematical Quantities, Then We Discussed That At Least $10^{\text {Th }}$ Order Dark Energy Is Required To Free And Stable Existence in Universe Then We Shows The Higher Order Dark Energy Of $11^{\text {th }}$ Order, Then There is Description of Cosmic Rays Shower of $E^{10+2 . n}$ And $E^{11+2 . n}$ Orders and higher level, At Two Ground Bases (i) $0.15012174857 *(10)^{18}$ And (ii) $4.465069415^{*}(10)^{18}$, Then Their Is Discussion About Contraction And Expansion Of Normal Matter Field By The Dark Energy At $14^{\text {th }}$ And $15^{\text {th }}$ Level. Then We Used The Kirchhoff's Radiation Law To explain the Unification of dark energy accepting and emitting bodies With The Dark Energy of universe Dark Energy. And the Absorption And Emitting Property of dark energy generator, Then The Stable State Of Systems For Uniting With The Universal Dark Energy.
\end{abstract}

Keywords: The ( $\boldsymbol{E} \boldsymbol{\beta} \boldsymbol{c})$ Equation, Kirchhoff Radiation Law, Dark Energy.

\section{Highlights-}

1. The Strange Behavior Of Particles And Matter To Interchange Their Varies Properties With Particle And Matter And Mathematical Quantities.

2. Shower Of Cosmic Rays.

3. Equations Of Varies Order Dark Energy.

4. Object's Unification with Universal Dark Energy.

5. Use of dark energy and dark matter.

\section{Article Outline:}

1. Introduction

2. Some Use full Transformation For Detecting Dark Energy.

3. Dark Energy According To Non-Interactive Mechanics And Prediction Of $10^{\text {th }}, 11^{\text {th }}, 12^{\text {th }}, 13^{\text {th }}$ Order Dark Energy And Shower Of Cosmic Rays With Two Bases At Ground Level.

4. Higher Order Expansion Of Dark Matter Of $14^{\text {th }}$ Order And Dark Energy (14 ${ }^{\text {th }}$ And $15^{\text {th }}$ order Dark Energy) Relations.

5. Stable Dark Energy.

6. The Impact Created By Dark Energy In Universe and The Entering Rule In Dark Energy Field.

7. Conclusion.

8. References.

\section{INTRODUCTION}

The Dark Energy is the most strange and mysterious power source in the universe. It is hard to categories the behavior of this power source but the most common behavior of this energy field is the expansion of universe in every possible way in total emptiness it expand in the manner that the expanded universe must hold the quality of stability so the destroyed or dying objects should be recreated this explain that this energy field holds the quality of natural intelligence , intelligence to hold the quality to destroy older objects , to recreate new objects for the continuity of universe, to expand universe with older memory and with new possibilities. The expansion of universe is the major work of dark energy, the maintains and expansion of universe is can't be possible until dark energy field holds the matter emitting field quanta or particles which hold the quality of matter emission, This type of quality is hold by the non intractable particles $(\mathrm{N}=-\mathrm{M})$, so here Non interactive mechanics will be used for explaining the behavior of dark energy field. The emitted matter convert into energy with motion and follow the curvature of space-time for create new objects, for expanding older objects, for destroy old age objects and to penetrate the boundaries of space-time in total emptiness for the expansion of universe to create new spacetime geometry which is free from periodic simulation this work is completed by the use of the properties of non intractable 
particles few of them are the velocity faster than light which is used to penetrate the older space-time structure and the property of matter emission which is used to fill the total nothingness of universe, this process becomes possible by following the geometry of the pre created space-time. According To General Theory Of Relativity Geometry Of Space Is Curved Or Follows Riemannian Geometry, According to Non-Interactive Mechanics Space Geometries Are Divided Into Two Types, According To the Flow Of Energies These Are

(a). Positive Curve Geometry (b). Negative Curve Geometry .

Positive curve geometry is the field where field quanta reside in its stable and ground energy state while the negative curve geometry is the field which participate in the material interaction and this is the place where the anti-particle of positive curve geometry exist in its ground state.

Statistically Positive Curve Geometry Is Cell Occupied Clockwise Rotating Geometry While Negative Curve is Neutral Cell Holding Anti-clockwise Rotating Geometry,

According Mass-Energy Relation E $\propto$ m, In Non-Interactive Mechanics We Have The Equation N + M =0 This Gives The Relation N= -M Here N Is Non-Intractable Particle And -M Is the Disintegrated Matter Which Has The Value Of $-\mathrm{M}=-$ $\frac{4 \pi V}{3 h^{3}} \cdot \frac{1}{4} \sqrt{\frac{8 m^{3} \pi}{\beta^{3}}} \cdot e^{\frac{\beta p^{2}}{2 m}}$.The Disintegrated Matter When Expanding, then opening the exponential term by binomial theorem, Then Considering, The First Term $-\mathrm{M}=-\frac{4 \pi V}{3 h^{3}} \cdot \frac{1}{4} \sqrt{\frac{8 m^{3} \pi}{\beta^{3}}}$. The Particles And Systems Come In This Negative Curve Geometry Are Called Beyond Disintegrated Matter (-M), That Means In This State Particles Doesn't Loss Their Atomic Weight While Action Is Going On.

Now If Considering The First Second Terms Both We Have.

$-\mathrm{M}=-\frac{4 \pi V}{3 h^{3}} \cdot \frac{1}{4} \sqrt{\frac{8 m^{3} \pi}{\beta^{3}}}-\frac{4 \pi V}{3 h^{3}} \cdot \frac{1}{4} \sqrt{\frac{8 m^{3} \pi}{\beta^{3}}} \cdot \frac{\beta p^{2}}{2 m}-\ldots$.

Under This stage Particle's Have Atomic Weight And They Loss Their Weight If They Get Divided.

As We Can See More We Enter In The Disintegrated Matter More We Loss The Atomic Mass Until The Condition Arrives When Rest Mass And Moving Mass Be Almost Zero And Gives A Stable State. Such As Photon, Graviton, NonIntractable Particle.

“So The Dark Energy Is The Energy Which Expand with The Negative Curve Geometry With The Rate Of $e^{\frac{\beta p^{2}}{2 m}}$.

\section{SOME USE FULL TRANSFORMATION FOR DETECTING DARK ENERGY}

After Reaching In The Dark Energy Field, Both Particle And Matter Behave Dramatically And Shows Varies Result. The Particle And Matter Relation Also Behaves Dramatically, in Dark Energy Field Strength And Communication Of Particle And Matter With Other Particles Increase Suddenly .In This Section We Will Study About These Forms Of Particle And Matter.

i. From The Equation Of Nishant Effect We Have

$\mathrm{E}^{n}=\frac{2(\mathrm{hVmax}-\mathrm{hVo})}{v^{2}} \cdot\left(g i e^{\alpha+\beta E i}-e^{-\alpha-\beta E i}\right)^{2 . n}$

For the Condition Where Non-Interactive Particle Is Not Present In The Matter Field $\mathrm{E}^{n}=\frac{2(\mathrm{hVmax})}{v^{2}} \cdot\left(-e^{-\alpha-\beta E i}\right)^{2 . n}$

Taking $\mathrm{n}=1$ And $\mathrm{E}=\mathrm{hv}$ On Left hand Side

And When We Have V App. Equal To C

$$
\mathrm{hV}=\frac{2(\mathrm{hV})}{C^{2}} \cdot\left(-e^{-\alpha-\beta E i}\right)^{2 . n}
$$

If We Have $\alpha$ And $\beta$ Constant Then

$C^{2}=2(-1)^{2 . n}$

For n Numbers Of System Where The Term $C^{2}$ On Left Hand Side Remains Constant

$C^{2}=2$ n. $(-1)^{2 . n}$

For $\mathrm{n}=1 / 2$ We Have

$C^{2}=2.1 / 2 \cdot(-1)^{\frac{2.1}{2}}$

$C^{2}=(-1)$

$\mathrm{C}=\sqrt{-1}$

$\mathrm{C}=\mathrm{i}$

ii. $\mathrm{E}^{n}=\frac{2(\mathrm{hVmax}-\mathrm{hVo})}{v^{2}} \cdot\left(g i e^{\alpha+\beta E i}-e^{-\alpha-\beta E i}\right)^{2 . n}$ 
AS $\mathrm{n}=1$ and $\mathrm{c}=\left(g i e^{\alpha+\beta E i}-e^{-\alpha-\beta E i}\right)$

$=\frac{2(\mathrm{hVmax}-\mathrm{hVo})}{v^{2}} \cdot(c)^{2}$

By photo electric effect

$1 / 2 \mathrm{~m} \mathrm{~V}^{\wedge} 2=\mathrm{h}$ vmax - hvo

Applying this gives

$\mathrm{E}=\mathrm{m} \cdot(c)^{2}$

By non-interactive mechanics

$\mathrm{N}=-\mathrm{M}$

Multiplying -1 on both sides gives

$-\mathrm{E}=-\mathrm{m} \cdot(c)^{2}$

$-\mathrm{E}=\mathrm{N} \cdot(c)^{2}$

$\mathrm{N}=\frac{-E}{c^{2}} \quad-\mathrm{P}$

Later

$\mathrm{E}=\mathrm{N}+\mathrm{M} .(c)^{2}$

M. $(c)^{2}=\mathrm{N}+\mathrm{M} .(c)^{2}$

$\mathrm{N}=0$

Putting in A gives

$0=\frac{-E}{c^{2}}$

As $0=\frac{N}{-M}$

Equating both equation gives

$\frac{N}{-M}=\frac{-E}{c^{2}}$

This gives

$\mathrm{N}=(M)^{2}-(\mathrm{b})$

iii. $\mathrm{N}=\frac{-E}{c^{2}} \quad-\mathrm{P}$

Substituting -(b) Into P We Have

$$
\begin{aligned}
& (M)^{2}=\frac{-E}{c^{2}} \\
& M^{2}=\frac{-\mathrm{m} \cdot(c)^{2}}{c^{2}} \\
& \mathrm{M}=-1 \quad-\mathrm{Q}
\end{aligned}
$$

iv. Substituting-Q In -(b)

We Have

$\mathrm{N}=(-1)^{2}$

$$
\mathrm{N}=1
$$

Putting $\mathrm{N}=1$ in $-(\mathrm{b})$ We Have

$(M)^{2}=1$

$$
\mathrm{M}= \pm 1
$$

For $\mathrm{M}=+1$ And Comparing This With Condition $\mathrm{N}=1$

We Have

$$
\mathrm{N}=\mathrm{M} \quad-(\mathrm{c})
$$

So The Results Are

i. $\mathrm{N}=\mathrm{M}$ ii. $\mathrm{N}=(M)^{2}$ iii. $\mathrm{N}= \pm 1$ iv. $\mathrm{N}=\mathbf{0}$ v. $\mathrm{M}= \pm 1$ vi. $\mathrm{M}=\mathbf{0}$ vii. $\mathrm{C}=\mathrm{i}$

The conditions for the use of above transformation.

i. Condition $\mathrm{N}=\mathrm{M}$, this condition is used only when the motion of objects are non relativistic.

ii. $N=(\mathrm{M})^{2}$, this condition is used when the two mass bodies are in the relativistic motion. 
iii. $\mathrm{N}= \pm 1$, this condition describe the emission of particle , $\mathrm{N}=+1$ for emission of non intractable particle beyond the field of the periodically simulating space time, and $\mathrm{N}=-1$ describe the emission of non intractable particle in periodically simulating space- time .

iv. $\mathrm{N}=0$ describes that non intractable particle is not present in the matter field.

v. $M= \pm 1$ describe the rate of matter-particle interaction and the direction of reaction $m=+1$ for the condition where total energy is less than the sum of kinetic and potential energy and $m=-1$ for the condition where total energy is higher then the sum of kinetic and potential energy. This condition is explained in the paper on the development of the photo electric effect.

vi. $\mathrm{M}=0$ this condition describe the emptiness of universe. Space- time which is not filled by matter.

vii. $\mathrm{C}=\mathrm{i}$ this condition is present when the non-intractable particle is not present in the matter field or the non intractable particle at rest, as the mass of non intractable particle and light are same but the velocities are different so for the condition when the non intractable particle either at rest or not present in the matter field the velocity of light becomes the highest in the matter field and the difference of velocity of light with other objects becomes as high that it becomes imaginary for all the objects moving relative to it.

These Results Shows The Dramatic Change in The Behavior Of Matter And Particles in the Dark Energy Field. Dark Matter and dark Energy Help To Interchange These Condition For Particles And Matter. Now We Use These Transformation To Get Minimum $10^{\text {th }}$ Order Dark Energy.

\section{DARK ENERGY ACCORDING TO NON-INTERACTIVE MECHANICS AND PREDICTION OF 10 ${ }^{\mathrm{TH}}, \mathrm{11}^{\mathrm{TH}}$ $\mathbf{1 2}^{\mathrm{TH}}, 13^{\mathrm{TH}}$ ORDER DARK ENERGY AND SHOWER OF COSMIC RAYS WITH TWO BASES AT GROUND LEVEL.}

In This Section We Will Study That At Least $10^{\text {Th }}$ Order Energy Is Required For Detecting Dark Energy In Universe. And The Cosmic Shower Of (E) $)^{\mathbf{1 0}+2 n}$ And(E) $)^{\mathbf{1 1 + 2 n}}$ Order.And Two Bases At Ground.

According To The Nishant Effect Of Non-Interactive Mechanics We Have Three Equations Which Are-

$$
\begin{aligned}
& \text { a. } \mathrm{E}^{n}=(\mathrm{N}+\mathrm{M}) \cdot\left(g i e^{\alpha+\beta E i}-e^{-\alpha-\beta E i}\right)^{2 . n} \\
& \text { b. } \mathrm{E}^{n}= \pm \sqrt{N^{2}+2 N M+(M)^{2}} \cdot\left(g i e^{\alpha+\beta E i}-e^{-\alpha-\beta E i}\right)^{2 . n} \\
& \text { c. } \mathrm{E}^{n}=\frac{2(\mathrm{hVmax}-\mathrm{hVo})}{v^{2}} \cdot\left(g i e^{\alpha+\beta E i}-e^{-\alpha-\beta E i}\right)^{2 . n}
\end{aligned}
$$

The Reason Behind To Multiplying These Equation Is That According To Nishant Effect Any Reaction In Universe Follow Tri-nature.

Now Multiplying All Three Equations Gives

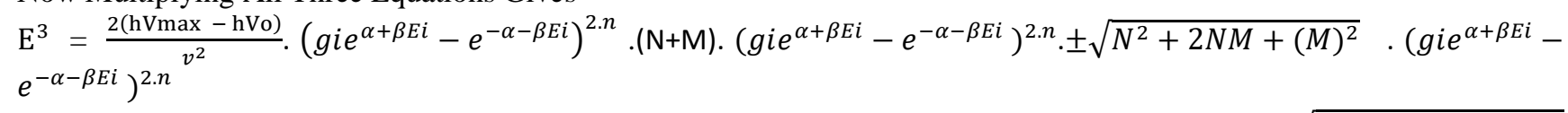

Taking hvmax $>$ hV0 And $\mathrm{n}=1$, gi $=0$ And According To Non-Interactive Mechanics $\mathrm{N}+\mathrm{M}= \pm \sqrt{N^{2}+2 N M+(M)^{2}}$ We Have

$$
\begin{aligned}
& \mathrm{E}^{3}=\frac{2(\mathrm{hVmax})}{v^{2}} \cdot(\mathrm{N}+\mathrm{M})^{2}\left(-e^{-\alpha-\beta E i}\right)^{6} \\
& \text { For } \mathrm{E}=\mathrm{hVmax} \text { and Then } \mathrm{E}=\mathrm{N}+\mathrm{M} \cdot(c)^{2} \\
& \mathrm{E}^{3}=\frac{2(E)}{v^{2}} \cdot(\mathrm{N}+\mathrm{M})^{2}\left(-e^{-\alpha-\beta E i}\right)^{6} \\
& \mathrm{E}^{3}=\frac{2 \mathrm{~N}+\mathrm{M} \cdot(c)^{2}}{v^{2}} \cdot(\mathrm{N}+\mathrm{M})^{2}\left(-e^{-\alpha-\beta E i}\right)^{6} \\
& \mathrm{E}^{3}=\frac{2 \cdot(c)^{2}}{(V)^{2}} \cdot(\mathrm{N}+\mathrm{M})^{3}\left(-e^{-\alpha-\beta E i}\right)^{6} \\
& \text { For }(c)^{2}>>(V)^{2} \quad, \frac{(c)^{2}}{(V)^{2}}=(c)^{2} \\
& \mathrm{E}^{3}=2(\mathrm{~N}+\mathrm{M})^{3} \cdot(c)^{2} \cdot\left(-e^{-\alpha-\beta E i}\right)^{6} \\
& \mathrm{E}^{3}=2(\mathrm{~N}+\mathrm{M})^{3} \cdot(c)^{2} \cdot\left(-e^{-\alpha-\beta E i}\right)^{6} \\
& \text { For } \mathrm{N}+\mathrm{M}=0 \text { And } 0=\frac{N}{-M} \\
& \mathrm{E}^{3}=2(0)^{3} \cdot(c)^{2} \cdot\left(-e^{-\alpha-\beta E i}\right)^{6} \quad-(\mathrm{X}) \\
& \mathrm{E}^{3}=2\left(\frac{N}{-M}\right)^{3} \cdot(c)^{2} \cdot\left(-e^{-\alpha-\beta E i}\right)^{6} \text { For } \mathrm{N}=-\mathrm{M} \\
& \mathrm{E}^{3}=2(1)^{3} \cdot(c)^{2} \cdot\left(-e^{-\alpha-\beta E i}\right)^{6} \\
& \text { Taking }\left(e^{-\alpha}\right)=\mathrm{Constant} \text { In Above Equation Then } \\
& \mathrm{E}^{3}=2(1)^{3} \cdot(c)^{2} \cdot\left(-e^{-\beta E i}\right)^{6} \\
& \mathrm{E}^{3}=2(1)^{3} \cdot(c)^{2} \cdot\left(-\left(1-\frac{6 h v}{k T}\right)\right)
\end{aligned}
$$




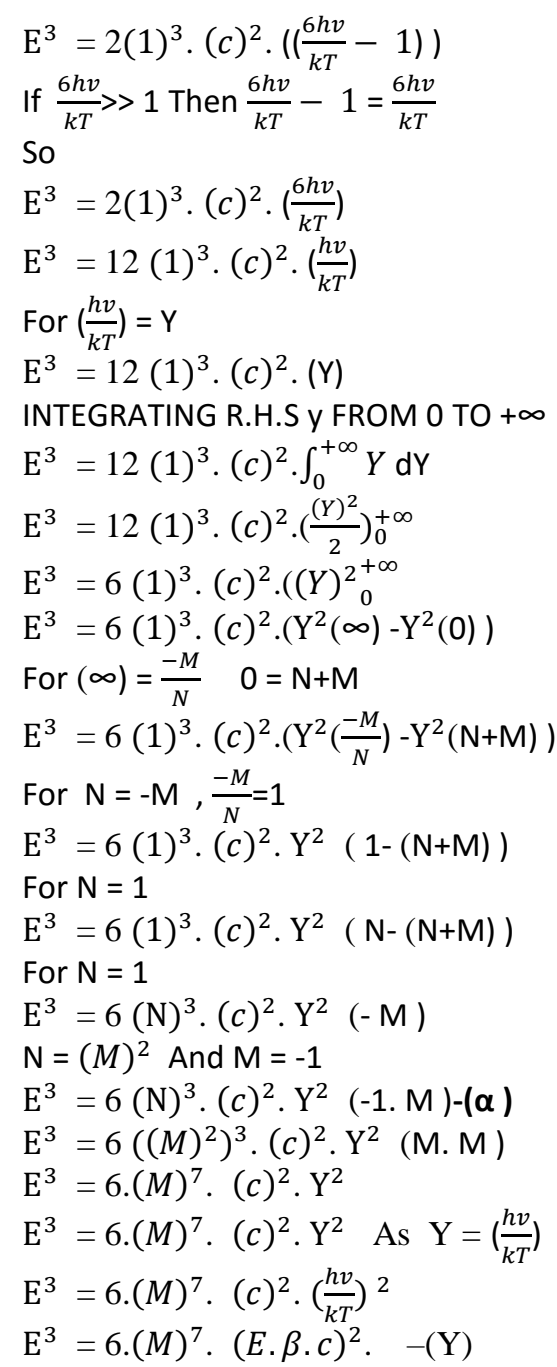

This Is the (E $\boldsymbol{\beta} \mathbf{c})$ Equation Of Dark Energy In Universe.

Now Moving Ahead As $\mathrm{M}=1$ So The Above Equation' Is

$\mathrm{E}^{3}=6 \cdot(M)^{7} \cdot(\text { E. } \beta . c)^{2} .1$

$\mathrm{E}^{3}=6 \cdot(M)^{7} \cdot(E \cdot \beta \cdot c)^{2} \cdot \mathrm{M}$

As We Are Talking About The Dark Energy Beyond -M So The Quantities Are Free From The Matter disintegration Properties.

$\mathrm{E}^{3}=6 \cdot(M)^{7} \cdot(E \cdot \beta)^{2} \cdot \mathrm{M} \cdot(c)^{2}$

$\mathrm{E}^{3}=6 \cdot(M)^{7} \cdot(E)^{3} \cdot(\beta)^{2}$

$\mathrm{E}^{3}=6 .(M)^{7} \cdot \mathrm{E} \cdot\left(\frac{h v}{k T}\right)^{2}$

$\mathrm{E}^{3}=6 .(M)^{7} \cdot \mathrm{E} \cdot\left(\frac{h v}{k T}\right)^{2} \cdot 1 \cdot \sqrt{-1} \cdot \sqrt{-1} \cdot \sqrt{-1} \cdot \sqrt{-1}$

$\mathrm{E}^{3}=6 \cdot(M)^{7}$.E. $\left(\frac{h v}{k T}\right)^{2}$. N .C.C.C.C $\quad$ As $\mathrm{N}=1$ And $\mathrm{C}=\sqrt{-1}$

After This $\mathrm{N}=(M)^{2}$ We Have

$\mathrm{E}^{3}=6 .(M)^{7} \cdot \mathrm{E} \cdot\left(\frac{h v}{k T}\right)^{2} \cdot(M)^{2} \cdot(C)^{4}$

$\mathrm{E}^{3}=6 \cdot(M)^{7} \cdot \mathrm{E} \cdot\left(\frac{h v}{k T}\right)^{2} \cdot(E)^{2}$.

$\mathrm{E}^{3}=6 \cdot(M)^{7} \cdot(E)^{3} \cdot\left(\frac{h v}{k T}\right)^{2}$

$\mathrm{E}^{3}=6 .(M)^{6} \cdot \mathrm{M} \cdot(E)^{3} \cdot\left(\frac{h c}{\Lambda k T}\right)^{2} \quad$ As $v=\frac{c}{\Lambda}$

$\mathrm{E}^{3}=6 \cdot(M)^{6} \cdot \mathrm{M} \cdot(C)^{2}(E)^{3} \cdot\left(\frac{h}{\kappa k T}\right)^{2}$

$\mathrm{E}^{3}=6 .(M)^{6} \cdot(E)^{4} \cdot\left(\frac{h}{\kappa k T}\right)^{2}$ 
Now As $\mathrm{C}=\mathrm{i}$ Then

$\mathrm{E}^{3}=6 .(E)^{4} \cdot\left(\frac{h}{k k T}\right)^{2} \cdot(M)^{6} \cdot \sqrt{-1} \cdot \sqrt{-1} \cdot \sqrt{-1} \cdot \sqrt{-1} \cdot \sqrt{-1} \cdot \sqrt{-1} \cdot \sqrt{-1} \cdot \sqrt{-1} \cdot \sqrt{-1} \cdot \sqrt{-1} \cdot \sqrt{-1} \cdot \sqrt{-1}$

$\mathrm{E}^{3}=6 .(E)^{4} \cdot\left(\frac{h}{\Lambda k T}\right)^{2} \cdot(M)^{6}$.C.C.C.C.C.C.C.C.C.C.C.C

$\mathrm{E}^{3}=6 \cdot(E)^{4} \cdot\left(\frac{h}{k k T}\right)^{2} \cdot(M)^{6} \cdot(C)^{12}$

$\mathrm{E}^{3}=6 \cdot(E)^{4} \cdot\left(\frac{h}{k k T}\right)^{2} \cdot(E)^{6}$

$\mathrm{E}^{3}=6 .(E)^{10} \cdot\left(\frac{h}{\kappa k T}\right)^{2}$

$\mathrm{E}^{3}=6 * 23.0539 *(10)^{-22}(E)^{10} \cdot\left(\frac{1}{\kappa T}\right)^{2}$

$\mathrm{E}^{3}=138.3234 *(10)^{-22} \cdot(E)^{10} \cdot\left(\frac{1}{K T}\right)^{2}$

$\mathrm{E}^{3}=1.383234 *(10)^{-24} \cdot(E)^{10} \cdot\left(\frac{1}{\kappa T}\right)^{2}$

$\mathrm{E}^{3}=1.38 *(10)^{-24} \cdot(E)^{10} \cdot\left(\frac{1}{\mathrm{KT}}\right)^{2}-(1 \mathrm{a})$

$0.7246376812 \times(10)^{24} \cdot \mathrm{E}^{3}=(E)^{10} \cdot\left(\frac{1}{K T}\right)^{2}-$

This value in Gev / $(C)^{2}$

$0.1501217487 \times(10)^{18} \cdot \mathrm{E}^{3}=(E)^{10} \cdot\left(\frac{1}{\kappa T}\right)^{2}-(1 \mathrm{c})$

For A Black Body $\Lambda=2 \mathrm{~L} / \mathrm{n} \quad \mathrm{n}=1+2+3+\ldots . .=-1 / 12$ And $\mathrm{L}=1$

$\Lambda=-24$ Unit

And If We Assume Temperature As The Difference Between The Energy Of Two Mass Bodies

$\mathrm{m} \mathrm{C}^{2}$

$\mathrm{m}_{C^{2}}$

Then $\mathrm{T}=2 \mathrm{~m} .(C)^{2}$ The Equation $-(1 \mathrm{a})$ Is

$\mathrm{E}^{3}=1.38 *(10)^{-24} \cdot(E)^{10} \cdot\left(\frac{1}{-24 * 2 m(C)^{2}}\right)^{2}$

As $\mathrm{M}=-1$

$\mathrm{E}^{3}=1.38 *(10)^{-24} \cdot(E)^{10} \cdot\left(\frac{1}{48(C)^{2}}\right)^{2}$

$\mathrm{C}=299792458 \mathrm{~m} / \mathrm{sec}$

$\mathrm{E}^{3}=0.0336226531 *(10)^{-24} \cdot(E)^{10}$

This value in Gev / $(C)^{2}$

$4.465069415^{*}(10)^{18} \mathrm{E}^{3}=(E)^{10}-(2)$

In above equation only R.H.S energy powers are changeable. Not the L.H.S energy powers as the $3^{\text {rd }}$ power always exist in universe.

This is The Required Dark Matter in Universe according To Non-Interactive Mechanics.

But Here The Condition Apply That N(Non-Interactive Particle) Is Always Present As N= +1 So29.7418528224

$*(10)^{24} \mathrm{E}^{3}=(E)^{10} * \mathrm{~N} \quad$ For $\mathrm{N}=(M)^{2} \quad$ And $\mathrm{C}=\mathrm{i} \quad$ We Have

$4.465069415 *(10)^{18} \mathrm{E}^{3}=(E)^{10 *}(M)^{2} * \mathrm{C}^{*} \mathrm{C} * \mathrm{C} * \mathrm{C}$

$4.465069415^{*}(10)^{18} \mathrm{E}^{3}=(E)^{12}$

$-(3)$

$4.465069415 *(10)^{18} \mathrm{E}^{3}=E^{10 *}(E)^{2 n} \quad$ Where n Is $1,2,3 \ldots \ldots$

$4.465069415 *(10)^{18} \mathrm{E}^{3}=(E)^{10+2 . n}$

Similarly-

$4.465069415^{*}(10)^{18} \mathrm{E}^{3}=(E)^{12+2 . n}$

For $\mathrm{n}=1 / 2,3 / 2,5 / 2 \ldots \ldots$

$4.465069415^{*}(10)^{18} \mathrm{E}^{3}=(E)^{11+2 . n}$

For $\mathrm{n}=1$ Equation $-(5)$ Is

$4.465069415^{*}(10)^{18} \mathrm{E}^{3}=(E)^{13}$

\section{So The Required Relation Of Dark Energy In Universe Are}

1. $\quad 0.15012174857 *(10)^{18} \cdot \mathrm{E}^{3}=(E)^{10} \cdot\left(\frac{1}{K T}\right)^{2}$

2. $4.465069415^{*}(10)^{18} \mathrm{E}^{3}=(E)^{10}$

3. $4.465069415 *(10)^{18} \mathrm{E}^{3}=(E)^{12}$

4. $4.465069415^{*}(10)^{18} \mathrm{E}^{3}=(E)^{12+2 . n}$

5. $\quad 4.465069415^{*}(10)^{18} \mathrm{E}^{3}=(E)^{11+2 . n}$ 


$$
4.465069415^{*}(10)^{18} \mathrm{E}^{3}=(E)^{13}
$$

These Are The $10^{\text {th }}, 11^{\text {th }}, 12^{\text {th }}, 13^{\text {th }}$ Order Dark Energy Relation.

Here We Can See That Eq. 1 Is The Eq. Of Dark Energy Related To inversely Square Of Temp. And Wavelength Dependent So When We Have Fewer Wavelength And Temperature Than There is More Possibility Of Finding Dark Energy.

But In Eq. 2-6 We Have Higher Order Dark Energy Which Temp. And Wavelength Free. SO The Dark Energy Of Eq. 2-6 Are Temperature And Wavelength Free. This isThe Reason Why Coldness Increases Of We Move From Earth To Neptune . And The CMBR Is Temperature Free State. So In Current Days We Have Detected Very Lower Level Dark Energies.

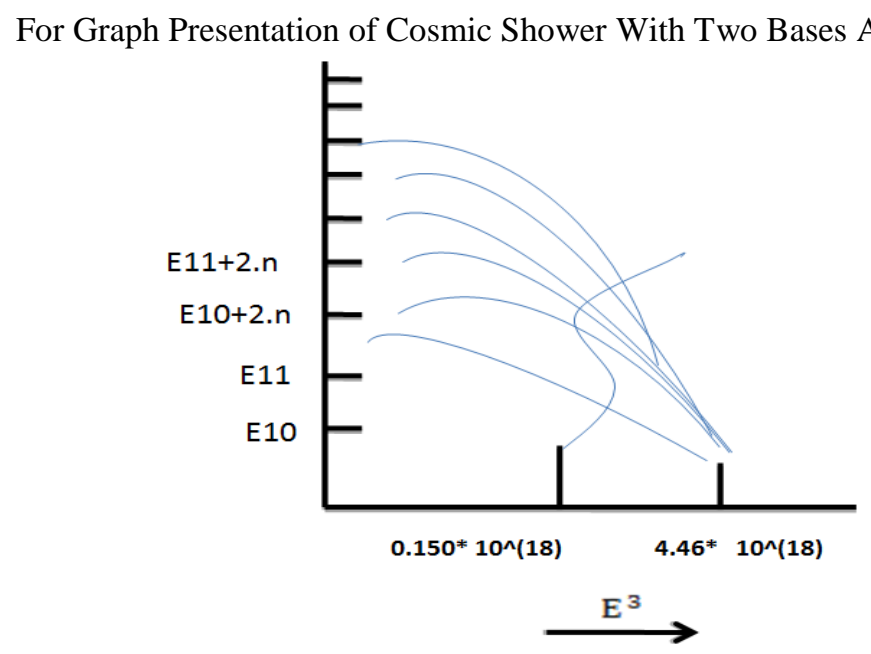

Fig. 1

\section{CREDIT : NISHANT KUMAR SHARMA}

In support of above graph some experimental proof of dark matter and its behavior is shown by BHASKAR DUTTA And LOUIS E. STRIGARI in their Paper 'Neutrino Physics with dark matter detectors' which is published in 'Annual Review of Nuclear and Particle Science'. Some Pictures of their paper are present. Here i want to clear this that my work and the experimental researches matched it is just a coincidence the above figure is far different with figures presented down their, both works are done in different times.

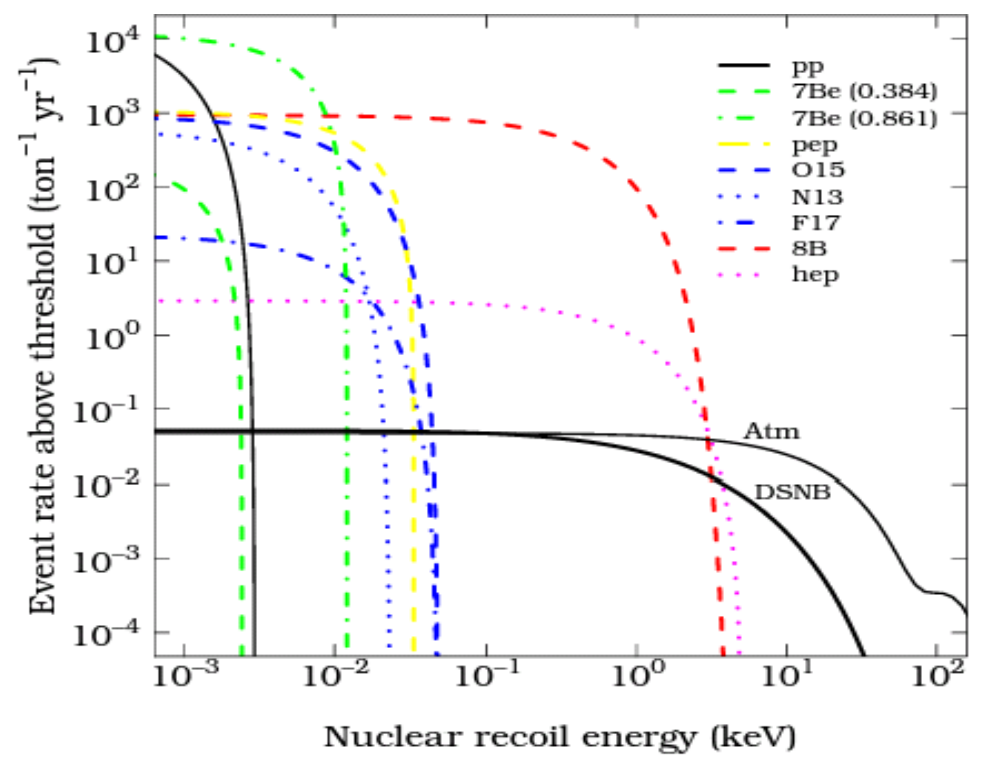

Figure 2

Solar, atmospheric, and diffuse supernova neutrino event rates above a threshold recoil energy. For the solar fluxes, each individual component is shown. For the atmospheric flux, shown is the sum of all four components

$\left(\nu_{e}, \bar{\nu}_{e}, \nu_{\mu}, \bar{\nu}_{\mu}\right)$. For the diffuse supernova neutrino fluxes, shown is the sum of all components $\left(\nu_{e}, \bar{\nu}_{e}, \nu_{\mu}, \bar{\nu}_{\mu}, \nu_{\tau}, \bar{\nu}_{\tau}\right)$. 


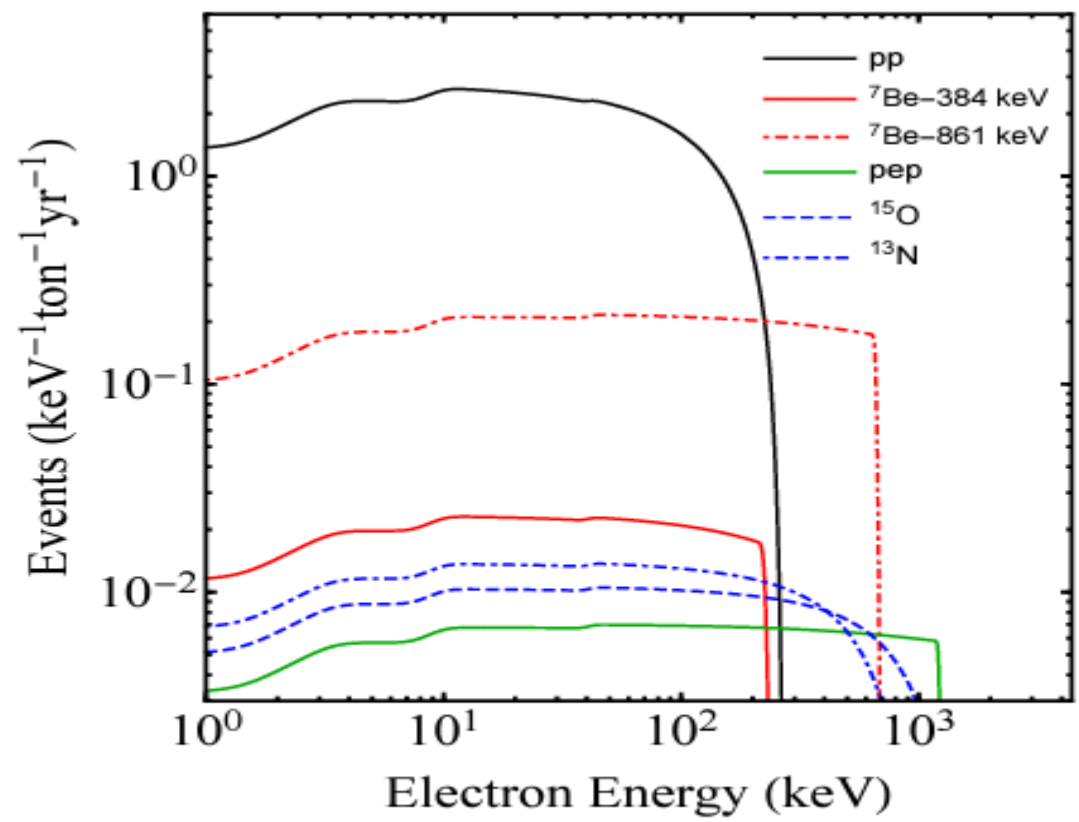

Figure 3

Electron recoil rates from solar neutrinos for a Xe detector. Figure reproduced from Ref. (41)

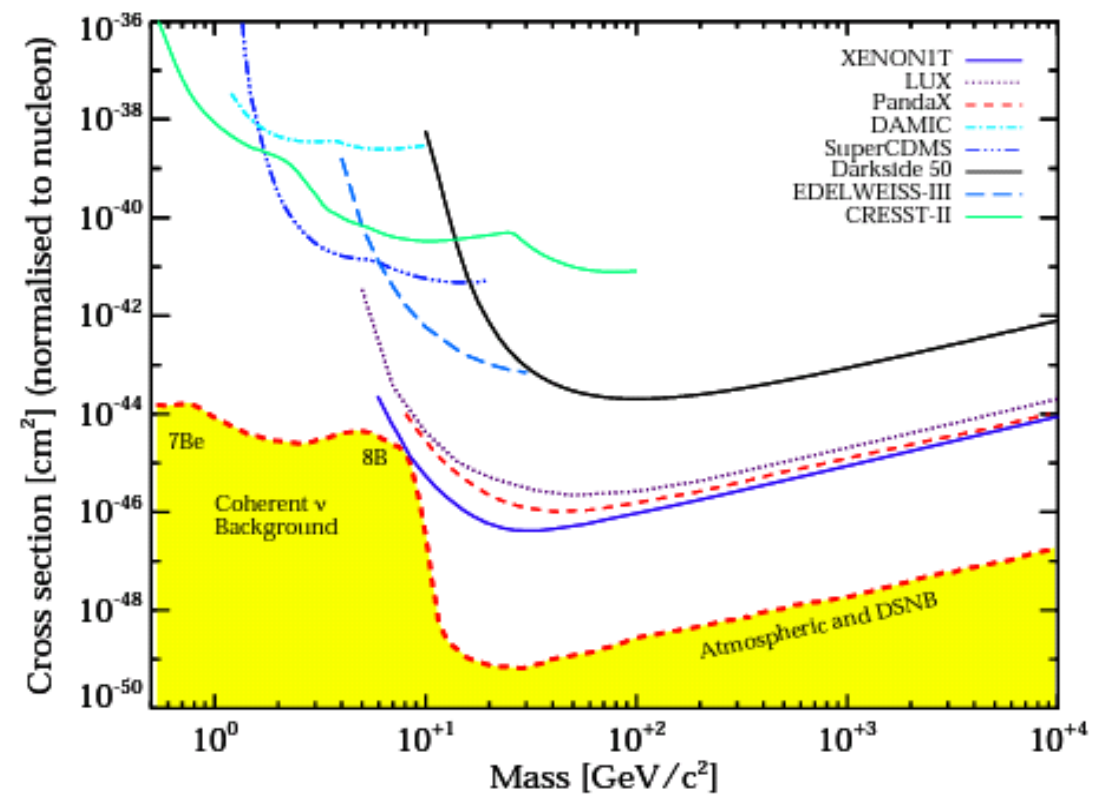

\section{Figure 4}

Exclusion limits on the dark matter-nucleon spin-independent scattering cross section from various direct dark matter detection experiments. The shaded region shows the "neutrino floor" as defined in Ref. (83)

\section{HIGHER ORDER EXPANSION OF DARK MATTER OF $14^{\mathrm{TH}}$ ORDER AND DARK ENERGY $\left(14^{\mathrm{TH}}\right.$ AND} $15^{\mathrm{TH}}$ ORDER DARK ENERGY) RELATIONS

Taking The Equation condition $\mathrm{E}^{3}=2(1)^{3} \cdot(c)^{2} \cdot\left(-e^{-\alpha-\beta E i}\right)^{6}$

Now Taking $\mathrm{N}=1$ And $\mathrm{N}=(M)^{2}$ Taking $\mathrm{C}=\left(\operatorname{gi} e^{\alpha+\beta E i}-e^{-\alpha-\beta E i}\right)$

$\mathrm{E}^{3}=2\left((M)^{2}\right)^{3} \cdot\left(\mathrm{gi} e^{\alpha+\beta E i}-e^{-\alpha-\beta E i}\right)^{2} \cdot\left(-e^{-\alpha-\beta E i}\right)^{6}$

For Negative Curve Geometry gi $=0$

$\mathrm{E}^{3}=2 .(M)^{6}\left(-e^{-\alpha-\beta E i}\right)^{2} \cdot\left(-e^{-\alpha-\beta E i}\right)^{6}$ 


$$
\mathrm{E}^{3}=2 .(M)^{6}\left(-e^{-\alpha-\beta E i}\right)^{8}
$$

Taking $e^{-\alpha}=$ Constant Then

$$
\begin{aligned}
& \mathrm{E}^{3}=2 \cdot(M)^{6}\left(-e^{-\beta E i}\right)^{8} \\
& \mathrm{E}^{3}=2 \cdot(M)^{6}\left(-e^{-\beta E i}\right)^{8} \\
& \mathrm{As} \mathrm{M}=-1 \\
& \mathrm{E}^{3}=2 \cdot(M)^{6}(-1)^{8} \cdot\left(e^{-\beta E i}\right)^{8} \\
& \mathrm{E}^{3}=2 \cdot(M)^{6}(M)^{8} \cdot\left(e^{-\beta E i}\right)^{8} \\
& \mathrm{E}^{3}=2 \cdot(M)^{14}\left(1-\frac{8 h v}{k T}\right) \\
& \mathrm{E}^{3}=2 \cdot(M)^{14}-\frac{16 h v}{k T}\left((M)^{14}\right)-(7)
\end{aligned}
$$

Our Condition of 14th order Dark Matter Complete.

But Looking An Another Case

$$
\begin{aligned}
& \mathrm{C}=\mathrm{i} \\
& \mathrm{E}^{3}=2 .(M)^{14}(C)^{28}-\frac{16 h v}{k T}\left((M)^{14}(C)^{28}\right) \text { as } \mathrm{E}=\mathrm{m}(C)^{2}
\end{aligned}
$$$$
\mathrm{E}^{3}=2 .(E)^{14}-\frac{16 E}{k T}\left((E)^{14}\right) \quad \text { As } \mathrm{E}=\mathrm{hv}
$$$$
\mathrm{E}^{3}=2 .(E)^{14}-\frac{16(E)^{15}}{k T}
$$$$
\mathrm{E}^{3}=2 .(E)^{14}-\frac{11.59 *(10)^{23}(E)^{15}}{T}
$$

If We Take Temperature As The Energy Difference Between Two Mass States Then $\mathrm{T}=2 \mathrm{~m}(C)^{2}$ Then

$$
\begin{aligned}
& \mathrm{m}_{C^{2}} \mathrm{~m}_{C^{2}} \\
& \mathrm{E}^{3}=2 .(E)^{14}-\frac{11.59 *(10)^{23}(E)^{15}}{2 \mathrm{~m}(C)^{2}}
\end{aligned}
$$

For $\mathrm{m}=0$

$\mathrm{E}^{3}=2 .(E)^{14}$

For $\mathrm{m}=+1$

$$
\begin{aligned}
& \mathrm{E}^{3}=2 .(E)^{14}-\frac{11.59 *(10)^{23}(E)^{15}}{2(C)^{2}} \\
& \mathrm{E}^{3}=2 .(E)^{14}-6.890606 \times(10)^{6}(E)^{15}
\end{aligned}
$$

For $\mathrm{m}=-1$

$$
\mathrm{E}^{3}=2 \cdot(E)^{14}+6.890606 \times(10)^{6}(E)^{15}
$$

So the required equations are

$$
\begin{array}{ll}
\text { 9. } & \mathrm{E}^{3}=2 .(E)^{14} \\
\text { 10. } & \mathrm{E}^{3}=2 .(E)^{14} \quad-6.890606 \times(10)^{6}(E)^{15} \\
\text { 11. } & \mathrm{E}^{3}=2 .(E)^{14}+6.890606 \times(10)^{6}(E)^{15}
\end{array}
$$

These are The Required Equation Of $14^{\text {th }}$ And $15^{\text {th }}$ Order Dark Energy Equation .

Above Equation Describe The Matter Behavior Of Every Object

'That During The Matter Contraction Process Any Matter Can't Go Lower Then 2. $(E)^{14}$ '.And During The Matter Expansion Process No Matter Can't Go Beyond 6.890606 $\times(10)^{6}(E)^{15}$.

All The Matter Act Between This Lowest And Highest Limit.

This Also Describe That Matter contract according to equation 8,

While During The Expansion Process Matter Expanded according to condition 9.

This Describes That In This Whole Process Matter Propagates In These Lowest To Highest Level In Form Of Wave or particle or both of representing field And To This Contraction \& Expansion Process A Field Particle Generates Which Should Remain Same As In Quantum, Classical And Relativistic Systems. This particle is Non Intractable Particle.

As We Mentioned In The Non-Intractable Mechanics That N-I Particles Are 20817.22m/sec Faster Than Normal Light but their masses are same .So Only This Field Generated N-i Particle Can Penetrate The Material Field. The difference between light and $\mathrm{N}$-I Particle is That the first generated particle is $\mathrm{N}-\mathrm{I}$ particle. The Identification

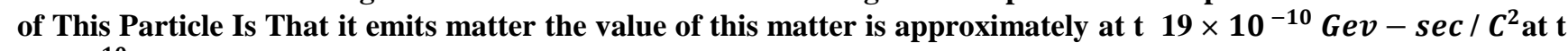
$=10^{-10}$ sec. 
Then second the light particle emit, with following simple harmonic motion and the energy of at $t 19 \times$ $10^{-10} \mathrm{Gev}-\mathrm{sec} / \mathrm{C}^{2}$ at $\mathrm{t}=10^{-9} \mathrm{sec}$ gather around the generating body.

\section{STABLE DARK ENERGY}

Taking Eq. $-(\alpha)$

$\mathrm{E}^{3}=6(\mathrm{~N})^{3} \cdot(c)^{2} \cdot \mathrm{Y}^{2}(-1 . \mathrm{M})$

Taking $\mathrm{M}=-1$ And $\mathrm{N}=(M)^{2}$ And $\mathrm{Y}=\mathrm{hv} / \mathrm{kT}=\mathrm{E} \beta$

$\mathrm{E}^{3}=6\left((M)^{2}\right)^{3} \cdot(c)^{2} \cdot(\mathrm{E} \beta)^{2}(\mathrm{M} . \mathrm{M})$

$\mathrm{E}^{3}=6 .(M)^{7}(\mathrm{E} \beta)^{2}\left(\mathrm{M} .(c)^{2}\right)$

$\mathrm{E}^{3}=6 .(M)^{7} \cdot(\mathrm{E} \beta)^{2}(\mathrm{E})$

$\mathrm{E}^{3}=6 .(M)^{7} \cdot(E)^{3} \cdot(\beta)^{2}$

$\mathrm{E}^{3}=6 \cdot(M)^{7} \cdot(E) \cdot\left(\frac{h v}{k t}\right)^{2}$

$\mathrm{E}^{3}=6 \cdot(M)^{7} \cdot(E) \cdot\left(\frac{h}{k T}\right)^{2} \cdot(V)^{2}$

$\mathrm{E}^{3}=6 \cdot(M)^{7} \cdot(E) \cdot\left(\frac{h}{k T}\right)^{2} \cdot\left(\frac{c}{\kappa}\right)^{2}$

For $\Lambda=2 \mathrm{~L} / \mathrm{n} \quad \mathrm{n}=1+2+3+\ldots . .=-1 / 12$ And $\mathrm{L}=1$

$\Lambda=-24$ Unit

$\mathrm{E}^{3}=6 \cdot(M)^{7} \cdot(E) \cdot\left(\frac{h}{k T}\right)^{2} \cdot\left(\frac{c}{-24}\right)^{2}$

$\mathrm{E}^{3}=\frac{1}{96} \cdot(M)^{7} \cdot(E) \cdot\left(\frac{h}{k T}\right)^{2} \cdot(\mathrm{C})^{2}$

For $\mathrm{N}=1$ And $\mathrm{N}=\mathrm{M}$ We Have

$\mathrm{E}^{3}=\frac{1}{96} \cdot(M)^{7} \cdot(E) \cdot\left(\frac{h}{k T}\right)^{2} \mathrm{~N} \cdot(\mathrm{C})^{2}$

$\mathrm{E}^{3}=\frac{1}{96} \cdot(M)^{7} \cdot(E) \cdot\left(\frac{h}{k T}\right)^{2} \mathrm{M} \cdot(\mathrm{C})^{2}$

$\mathrm{E}^{3}=\frac{1}{96} \cdot(M)^{7} \cdot(E) \cdot\left(\frac{h}{k T}\right)^{2} \cdot \mathrm{E}$

$\mathrm{E}^{3}=\frac{1}{96} \cdot(M)^{7} \cdot(E)^{2} \cdot\left(\frac{h}{k T}\right)^{2}$

Adding $(E)^{2}=(m)^{2} \cdot(C)^{4}$ As $\mathrm{N}=1, \mathrm{~N}=\mathrm{M}$ And $\mathrm{C}=\mathrm{I}$ Above Equation Becomes

$\mathrm{E}^{3}=\frac{1}{96} \cdot(M)^{7} \cdot(E)^{4} \cdot\left(\frac{h}{k T}\right)^{2}$

$1=\frac{1}{96} \cdot(M)^{7} \cdot \mathrm{E} \cdot\left(\frac{h}{k T}\right)^{2}$

If We Take Temperature As The Energy Difference Between Two Mass States Then $\mathrm{T}=2 \mathrm{~m}(C)^{2}$

$\mathrm{m} \mathrm{C}^{2}$

$\mathrm{m} C^{2}$

Then

$1=\frac{1}{96} \cdot(M)^{7} \cdot \mathrm{E} \cdot\left(\frac{h}{k}\right)^{2} \cdot\left(\frac{1}{T}\right)^{2}$

$1=\frac{1}{96} \cdot(M)^{7} \cdot \mathrm{E} \cdot\left(\frac{h}{k}\right)^{2} \cdot\left(\frac{1}{2 \mathrm{~m}(C)^{2}}\right)^{2}$

$1=\frac{1}{96} \cdot(M)^{7} \cdot \mathrm{E} \cdot\left(\frac{h}{k}\right)^{2} \cdot\left(\frac{1}{4(m)^{2}(C)^{4}}\right)$

$1=\frac{1}{386} \cdot(M)^{7} \cdot\left(\frac{h}{k}\right)^{2} \cdot\left(\frac{1}{(m)^{2}(C)^{4}}\right)$

Taking $\mathrm{C}=\mathrm{i}$ We Have

For $\mathrm{N}=1$ and $\mathrm{N}=\mathrm{M}$

$$
1=\frac{1}{386} \cdot(M)^{5} \cdot\left(\frac{h}{k}\right)^{2} \cdot 1
$$

$$
1=\frac{1}{386} \cdot(M)^{6} \cdot\left(\frac{h}{k}\right)^{2}
$$

For $\mathrm{N}=\mathrm{M}$ and $\mathrm{N}=1$

$$
\begin{aligned}
& 1=\frac{1}{386} \cdot(N)^{6} \cdot\left(\frac{h}{k}\right)^{2} \\
& 1=\frac{1}{386} \cdot\left(\frac{h}{k}\right)^{2} \\
& 386(k)^{2}=(h)^{2}
\end{aligned}
$$




$$
\begin{aligned}
1=\frac{1}{386} & \cdot\left(\frac{6.626 *(10)^{-34}}{1.38 *(10)^{-23}}\right)^{2} \\
1 & =\frac{1}{386} \cdot\left(4.801 *(10)^{-11}\right)^{2} \\
1 & =0.0597251688 *(10)^{-22} \\
1 & =0.0597251688 *(10)^{-22} \text { Kelvin-Sec }
\end{aligned}
$$

Now Using Kirchhoff's Radiation Law

'The Object Should Receive0.0597251688*(10) $)^{-22}$ Kelvin- Sec Temperature To Unite With The Universal Dark Energy'

Also

$16.7433599618 *(10)^{22}(K)^{-1} \cdot(\text { Sec })^{-1}=1 \quad-(\mathrm{y})$

'The Object Should Emit16.7433599618 * $(10)^{22}(K)^{-1}(S e c)^{-1}$ Temperature To Unite With Universal Dark Energy'.

So 'The Object Should Receive0.0597251688*(10)-22 Kelvin- Sec Temperature and The Object Should Emit16.7433599618* $(10)^{22}(K)^{-1}(S e c)^{-1}$ Temperature To Unite With Universal Dark Energy'.

After Reaching These Conditions Every Field Quanta Get Stable State In Natural And Ordinary Material World.

6.The Impact Created By Dark Energy In Universe and The Entering Rule In Dark Energy Field -

Taking the Above equation condition

$1=0.0597251688 *(10)^{-22}$ Kelvin - Sec

This can be written as

$1=0.0597251688 *(10)^{-22}(M)$ Kelvin - Sec , where $\mathrm{M}=1$, For total energy is less than the sum of kinetic and potential energy

$1=(-11.0229152797)(M)$ Kelvin - Sec

For 1 kelvin-sec

$1=(-11.0229152797)(M)$

$1=(-11.0229152797) \mathrm{M}$

( 11.0229152797) $\mathrm{M}=-1$

This is the Entering condition in dark Energy Field .

According to Non - Interactive Mechanics

$12 \mathrm{M}=-1$

this is the Required Condition for the entering of Non Intractable Particles in Matter field Known As Ramanujan Entering For particle following Positive curve Geometry.

While on other side Dark Energy Field Required (11.0229152797) M = - 1, Condition to Enter in the Dark Energy Field .

This is why No Ordinary Matter Particle Could Enter in Dark Energy Field. And If we try to Enter in the Dark Energy Field With the Condition 12M =-1 then Dark Energy Field will Repulse us. Which is same as experimental and Theoretical prediction.

The difference in Ramanujan Entering and dark energy condition is 0.9770847203 M This is The Free Matter and Purest Matter. Which remains during the movement of dark matter and dark energy or we can say That a object losses this mass value in the process of moving with dark energy, This remaining matter convert as dark matter.

So By Following Dark Energy objects losses their Normal matter which is only be regain by the infinite expansion property of Non Intractable Particles.

As Also The difference of both condition is the reason of ORIGINATION OF LIFE AND CONSIOUSNESS in the universe.

After Achieving this state the only work is left for dark energy and dark matter is to circulate the periodic quantum and material simulation world.

\section{CONCLUSION}

Non-Interactive Mechanics Is A Very Useful Tool For Detecting Dark Matter And Dark Energy In The Universe ,It describes The Interchange Property Of Matter, Particle And Imaginary Form Of Light At Dark Energy Field. It Describes The Classification Of The Dark Matter And Dark Energy In Different Orders, Here We Can See That Eq. 1 Is The Eq. Of Dark Energy Related To inversely Square Of Temp. And Wavelength Dependent So When We Have Less Wavelength And Less Temperature We Have More Possibility Of Finding Dark Energy.

But In Eq. 2-6 We Have Higher Order Dark Energy Which Temp. And Wavelength Free. SO The Dark Energy Of Eq. 2-6 Are Temperature And Wavelength Free. This Is The Reason Why Coldness Increases Of We Move From Mercury To Neptune. And The Pure CMBR Is Temperature Free State, In Current Days We Have Very Lower Level Dark Energies. Then It Describes The Two Bases Of Cosmic Ray Shower. 
Then The Expansion And Contraction Of Material Field By $14^{\text {th }}$ And $15^{\text {th }}$ Order Dark Energy And Movement Of NonIntractable Particle In This Systems Quantum, Classical And Relativistic Systems.

Then It Describes That When We Have Dark by Following Kirchhoff Law In Temperature Emitting And Receiving Form, It reach To It's Purest Form And Combined With The Universal Dark Energy. At last we describe that why it is hard to Enter in the Dark Energy Field because the entering rule in common matter field dark energy field are different ,this is why dark Energy and dark matter repulse those objects which try to Enter in it.

So for detecting dark Energy and dark matter we must follow its entering rule , the absorption and emitting property for stable dark energy state and then following relation 1 to 11 to achieve limitless and clean Energy.

\section{REFERENCES}

[1] Nishant Kumar Sharma, Non-Interactive Mechanics, Journal Of Nuclear And Particle Physics, Vol.9 No.1, 2019, pp. 18-41. doi:10.5923/j.jnpp.201909901.03.

[2] Satya Prakash, "statistical Mechanics", KNRN, 2015.

[3] Kumar,V : Gupta,S.L.," Elementary Statistical Mechanics", Pragati Prakashan , 2017

[4] Bhaskar Dutta, Louis E. Strigari ,Neutrino Physics with dark matter detector, Annual Review of Nuclear and Particle Science , vol. 69, pp. 137-161, doi : 10. 1146, annurev-nucl-101918-023450

\section{AUTHOR PROFILE}

Mr. Nishant Kumar Sharma is B.sc,, M.sc . in. Physical Science from Kumaun University in 2015, 2017. $\mathrm{He}$ is currently working as Independent Researcher and Published Two Articles in international journals which unifies Classical ,Relativistic and quantum mechanics, the papers Are Non Interactive mechanics, Law of gravitation for Non Intractable Particles . Article Law of gravitation for Non Intractable Particles is published in ISROSET Journal IJSRPAS. 